\title{
How to Measure Loneliness during COVID-19: Development of a New Scale
}

\author{
Gazzier Muhammad
}

Psychology Research Center

\begin{abstract}
This research report develops a new scale of loneliness for COVID-19. It discusses three existing measures of loneliness, addresses their limitations, and proposes new ways to mitigate the limitations, especially in the context of COVID-19. A three-question scale with Likert-scale is developed for COVID-19 crisis, answering the call for better measurement strategy in the clinical psychology of COVID-19.
\end{abstract}

Keywords: loneliness, COVID-19, measurement, scale

\section{Introduction}

Because loneliness is listed as a unique psychological challenge of the COVID-19 or coronavirus crisis, psychologists and psychiatrists alike will have to measure it during the crisis. This article reviews how loneliness is measured in the extant literature, lists the potential pitfalls of these measures, and propose a new measure that is more appropriate, in our opinion, to validly and reliably measure loneliness.

\section{Limitations of Existing Scale}

Previous research calls for validation of the loneliness measure ( $\mathrm{Li}$ and Wang, 2020b). We believe the first step is to take stock of what is available to us. 
The first widely adopted measure is the UCLA Loneliness measure (Russell 1996). Results show that it is a highly reliable (Russell, 1996). The original version of UCLA Loneliness Scale consists of 20 questions. It is arguable too long for telephone-based survey, which is widely used during the crisis. Also, if people are already lonely, how would they be psychologically strong to answer all these questions? These concerns make the measure less acceptable for COVID-19 researchers. We disagree with the call to use the "full-scale" UCLA Loneliness measure.

Another possibility is the adapted measure used in English Longitudinal Study of Ageing (ELSA). This measure uses the three-question short form of the UCLA Loneliness measure. The questions are: "How often do you feel you lack companionship", "How often do you feel left out" and "How often do you feel isolated from others" (Pikhartova, Bowling \& Victor, 2016). The problem with this measure is that in a 9-point Likert scale, the upper quartile is classified as lonely. The other quartiles are classified as "not lonely" (Steptoe, Shankar, Demakakos, \& Wardle, 2013). We believe this binary classification is arbitrary because loneliness often develops gradually, so minor loneliness is also important to be considered.

Finally, Li and Wang (2020a) uses a one-question measure of loneliness. The strength of their way of measuring is that they include degrees of loneliness instead of treating it as a binary outcome. They admit that it must be validated. We believe that for such a long survey (UKHLS COVID-19) which they use, it is quite understandable that only one question can be devoted to loneliness. However, we believe that a few more questions will increase validity of the measure.

\section{Development of New Scale}

We argue that a combination of the three-questions used in ELSA (Pikhartova, Bowling \& Victor, 2016) and the continuous scale used in Li and Wang (2020)'s study is the best way to measure loneliness during the COVID-19 crisis. 
Table 1: A New Scale for Loneliness during COVID-19

\begin{tabular}{|c|c|c|c|c|c|}
\hline & $\begin{array}{l}\text { Strongly } \\
\text { disagree }\end{array}$ & Disagree & $\begin{array}{l}\text { Neither } \\
\text { agree nor } \\
\text { disagree }\end{array}$ & Agree & $\begin{array}{l}\text { Strongly } \\
\text { agree }\end{array}$ \\
\hline $\begin{array}{l}\text { How often do you feel you } \\
\text { lack friendship }\end{array}$ & 1 & 2 & 3 & 4 & 5 \\
\hline $\begin{array}{l}\text { How often do you feel } \\
\text { isolated from others }\end{array}$ & 1 & 2 & 3 & 4 & 5 \\
\hline $\begin{array}{l}\text { How often do you feel left } \\
\text { out }\end{array}$ & 1 & 2 & 3 & 4 & 5 \\
\hline
\end{tabular}

Note: questions adapted from three-question UCLA Loneliness Scale

We suggest that a score greater than 10 out of 15 is considered threshold for loneliness, following previous research on this concept (Pikhartova, Bowling \& Victor, 2016). However, it is worth repeating again that a continuous loneliness score is better than the binary division. Therefore, the research using the scale should consider model specification for continuous outcomes. In some cases, ordered-probability model can also be specified if the assumptions are met.

Future research could follow our suggestion to validate on this innovative measure. Of particular importance is to validate it in a large population, because loneliness is considered a pandemic in the crisis.

\section{Conclusion}

The paper discusses the existing measure of loneliness, addresses their limitations, and proposes ways to mitigate the limitations, especially in the context of COVID-19. It develops a new scale 
to measure loneliness for researchers to use. Future research needs to validate the scale to establish its validity and reliability in a population sample.

\section{References:}

Jitka Pikhartova, Ann Bowling \& Christina Victor (2016) Is loneliness in later life a selffulfilling prophecy? Aging \& Mental Health, 20:5, 543-549, DOI: $10.1080 / 13607863.2015 .1023767$

Li, Lambert Zixin, and Senhu Wang. (2020) Prevalence and Predictors of General Psychiatric Disorders and Loneliness during COVID-19 in the United Kingdom: Results from the Understanding Society $\quad$ UKHLS. medRxiv. https://doi.org/10.1101/2020.06.09.20120139

Li, Lambert Zixin, and Senhu Wang. (2020) Prevalence and Predictors of General Psychiatric Disorders and Loneliness during COVID-19 in the United Kingdom. Psychiatry Research, 291, 113267. DOI: https://doi.org/10.1016/j.psychres.2020.113267.

Russell, Daniel W. (1996) UCLA Loneliness Scale (Version 3): Reliability, validity, and factor structure. Journal of personality assessment 66.1 (1996): 20-40.

Steptoe, A., Breeze, E., Banks, J., \& Nazroo, J. (2012). Cohort profile: The English Longitudinal Study of Ageing. International Journal of Epidemiology. doi:10.1093/ije/dys168 